\title{
Climbing the rotational ladder to chirality
}

\author{
Alec Owens, ${ }^{1,2}$ Andrey Yachmenev, ${ }^{1,2, *}$, Sergei N. Yurchenko, ${ }^{3}$ and Jochen Küpper ${ }^{1,2,4}$ \\ ${ }^{1}$ Center for Free-Electron Laser Science, Deutsches Elektronen-Synchrotron DESY, Notkestraße 85, 22607 Hamburg, Germany \\ ${ }^{2}$ The Hamburg Center for Ultrafast Imaging, Universität Hamburg, Luruper Chaussee 149, 22761 Hamburg, Germany \\ ${ }^{3}$ Department of Physics and Astronomy, University College London, Gower Street, WC1E 6BT London, United Kingdom \\ ${ }^{4}$ Department of Physics, Universität Hamburg, Luruper Chaussee 149, 22761 Hamburg, Germany
}

(Dated: October 2, 2018)

\begin{abstract}
Molecular chirality is conventionally understood as space-inversion-symmetry breaking in the equilibrium structure of molecules. Less well known is that achiral molecules can be made chiral through extreme rotational excitation. Here, we theoretically demonstrate a clear strategy for generating rotationally-induced chirality (RIC): An optical centrifuge rotationally excites the phosphine molecule $\left(\mathrm{PH}_{3}\right)$ into chiral cluster states that correspond to clockwise $(R$-enantiomer $)$ or anticlockwise $(L-$ enantiomer) rotation about axes almost coinciding with single $\mathrm{P}-\mathrm{H}$ bonds. Application of a strong $\mathrm{dc}$ electric field during the centrifuge pulse favors the production of one rotating enantiomeric form over the other, creating dynamically chiral molecules with permanently oriented rotational angular momentum. This essential step toward characterizing RIC promises a fresh perspective on chirality as a fundamental aspect of nature.
\end{abstract}

Chiral molecules exist in two structural forms known as enantiomers, which are the mirror image of each other and thus non-superimposable by translation and rotation. The wavefunctions of the enantiomers have no definite parity, i. e., space-inversion symmetry, as they tunnel periodically from one structure to the other on a timescale determined by the energy barrier separating the two enantiomeric forms. Molecular chirality, in the traditional sense, is associated with the barriers formed by the static potential energy surface, typically so large that the enantiomers do not readily interconvert at room temperature and can be separated by chemical means and stored. There is, however, the possibility to induce and control chirality in achiral molecules through extreme rotational excitation into states where centrifugal distortions produce equivalent asymmetric structures separated by high kinetic energy barriers [1]. The appearance of such chiral high angular momentum states is closely linked to the energetic clustering of rotational states, an effect displayed by certain polyatomic molecules exhibiting local mode vibrations [2]. Given the importance of chirality to our understanding of molecular and material behavior, the ability to create chirality in achiral systems is of great fundamental interest. For example, it has recently been shown that isotopic substitution can induce chirality in achiral molecules and catalyze asymmetric reactions [3, 4].

Very little is known about the phenomenon of rotationally-induced chirality (RIC), but ultrashort-pulse strong-field laser physics has brought new impetus to the preparation of molecules in highly excited rotational states. The main obstacle to overcome when generating large amounts of rotational excitation is that selection rules permit only small changes in the overall angular momentum $J$. With the development of clever adiabatic and non-adiabatic approaches [5, 6], this is now far less challenging and it is possible to efficiently create rotational wavepackets with a narrow and well-defined distribution of states. Molecular superrotors, as they are known in the literature, display novel behavior. For example, they are far more resistant to collisions and reorientation [7-4] making them interesting objects for scattering [10, spectroscopy 11, and dynamics 12. Superrotors provide an ideal testing ground for exploring RIC, and by learning to control and characterize this dynamic effect, e.g., the optical activity, there is an opportunity to gain new insights into chirality, specifically motion-dependent chiral systems and the nature of their interactions.

In this work, a clear strategy for producing dynamically chiral molecules through extreme rotational excitation is presented. The scheme is outlined in Fig. 1 and realized through robust quantum mechanical simulations of RIC in the phosphine molecule $\left(\mathrm{PH}_{3}\right) . \mathrm{PH}_{3}$ is initially considered in its ground rovibronic state as a symmetric top molecule possessing $\mathbf{C}_{3 v}(\mathrm{M})$ molecular symmetry; inversion tunneling has a high barrier and is neglected. An optical centrifuge [13, which is a non-resonant, linearly polarized laser pulse that undergoes accelerated rotation about the direction of propagation, is used to adiabatically spin phosphine into highly excited rotational states. Above the critical value of $J_{\mathrm{c}} \approx 35$, the rovibrational energy levels of $\mathrm{PH}_{3}$ form sixfold degenerate clusters [14, which classically can be interpreted as a "racemic mixture" of clockwise $(R)$ and anticlockwise $(L)$ rotations about localization axes approximately coinciding with a $\mathrm{P}-\mathrm{H}$ bond, see Fig. 2. Due to strong centrifugal coupling, three dynamic and symmetrically equivalent molecular structures emerge, where one of the $\mathrm{P}-\mathrm{H}$ bonds about which the molecule rotates, is shorter than the other two. The three asymmetrical structures can rotate in either a clockwise or anticlockwise direction. They are energetically indistinguishable from one another and separated by high kinetic energy barriers, which is analogous to conventional "static" enantiomers with potential barriers. If $\mathrm{PH}_{3}$ is prepared in a state corresponding to a preferred 


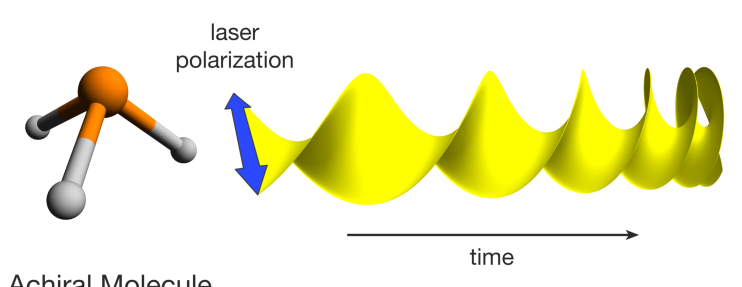

Achiral Molecule

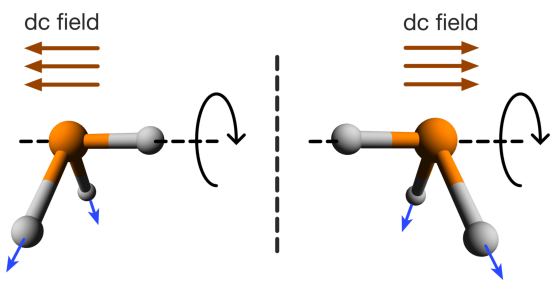

Rotating Enantiomers

FIG. 1. Rotationally-induced chirality in $\mathrm{PH}_{3}$ using an optical centrifuge in conjunction with a static electric field. Reversing the direction of the dc field produces the other rotating enantiomer.

direction of rotation in the laboratory frame, the permutation symmetry is broken by centrifugal distortions, whilst parity is broken by unidirectional rotation. The result is a rotating enantiomer, where clockwise rotation corresponds to the $R$-enantiomer, and anticlockwise rotation to the $L$-enantiomer.

The relationship between the localized chiral cluster states and the delocalized wavefunctions in the $\mathbf{C}_{3 v}(\mathrm{M})$ symmetry group is shown in Fig. 2. It is evident that any interaction of the permanent molecular electric dipole moment with a strong electric field will mix the delocalized wavefunctions of quasi-degenerate states, e.g., $A_{1}$ with $A_{2}$, in such a way that combinations of states dominated by either $R$ or $L$ localized states are generated. Hence, by applying a strong dc field during the centrifuge pulse either one of the rotating enantiomers can be exclusively produced depending on the direction of the field. This is an essential step toward characterizing RIC as each enantiomer can be isolated for further investigation. Furthermore, it demonstrates that an ensemble of dynamically chiral molecules with "permanently" oriented rotational angular momentum can be created, where the orientation of the angular momentum with respect to the $\mathrm{P}-\mathrm{H}$ bond, about which rotation takes place, defines the rotating enantiomer. That is, the difference between the centrifuge with and without the dc field is the orientation of the angular momentum in the molecular frame.

It should be noted that the Raman-type excitation by the optical centrifuge couples states with the same symmetry, whereas the dc field couples states whose productsymmetry spans the symmetry of the dipole moment $A_{2}$, i. e., $A_{1} \leftrightarrow A_{2}$ and $E \leftrightarrow E$. Therefore, depending on the symmetry of the initial rotational state, only a pair of $A_{1}$ and $A_{2}$ or a pair of $E^{(1)}$ and $E^{(2)}$ delocalized components of the rotational cluster will give rise to the effect of RIC.

The theoretical approach is described in detail in the supplemental material 15. Numerical simulations were fully quantum mechanical and considered all major ground-state electronic correlation within the BornOppenheimer approximation, nuclear motion, and external field effects to an accuracy capable of supporting high-resolution spectroscopic measurements, i. e., sub$\mathrm{cm}^{-1}$ accuracy for vibrational and $10^{-4}-10^{-5} \mathrm{~cm}^{-1}$ for rotational energies. The stationary rovibrational energies and eigenfunctions of $\mathrm{PH}_{3}$ up to $J=60$ were computed by direct numerical variational calculations [16-18] from an empirically refined, six-dimensional potential energy surface 19. To describe external field effects, the matrix elements of the electric dipole moment and electric polarizability tensor were evaluated in the basis of rovibrational states. In quantum dynamics simulations, which employed the computer program RichMol [20, the timedependent wavefunction was built from a superposition of rovibrational eigenfunctions with the time-dependent coefficients obtained by numerical solution of the timedependent Schrödinger equation using the split-operator method.

An optical centrifuge with maximum intensity $\epsilon_{0}=$ $5.2 \times 10^{13} \mathrm{~W} / \mathrm{cm}^{2}$ and chirp rate $\beta=(2 \pi c)^{2} \cdot 25 \mathrm{~cm}^{-2}$ was applied along the laboratory-fixed $Z$ axis for a duration of $t=36.8 \mathrm{ps}$. Simulations were started in the rovibrational ground state $(J=k=m=0)$, corresponding to samples that can be produced from cold beams using the electric deflector [21, 22]. The centrifuge field aligns the most polarizable molecular axis through the interaction with the induced molecular dipole moment, trapping and forcing the molecule to follow the rotating polarization. $\mathrm{PH}_{3}$ is predominantly excited via $\Delta J=2, \Delta m=-2$ rotational Raman transitions through the highest energy state of each $J$ value. Here, the quantum numbers $k$ and $m$ correspond to the projection, in units of $\hbar$, of $J$ onto the molecule-fixed $z$ axis and laboratory-fixed $Z$ axis, respectively. For calculations involving a static electric field, a field strength of $1 \mathrm{MV} / \mathrm{cm}$ [23] was applied along the laboratory-fixed $Z$ axis.

The phenomenon of RIC is well illustrated with the chosen laser parameters, but the centrifuge intensity $\epsilon_{0}$ is expected to cause ionization of $\mathrm{PH}_{3}$. However, the same conclusions can still be reached with lower optical and static field strengths, and when using an experimentally realistic pulse envelope for the optical centrifuge, see the supplemental material [15. It should be noted that because $\mathrm{PH}_{3}$ is highly toxic, we anticipate that future experiments will be performed on other systems, most likely heavier rotors, which require weaker optical and static field strengths, allowing for the practical realization 


\section{a. Clustering of rotational energy levels}

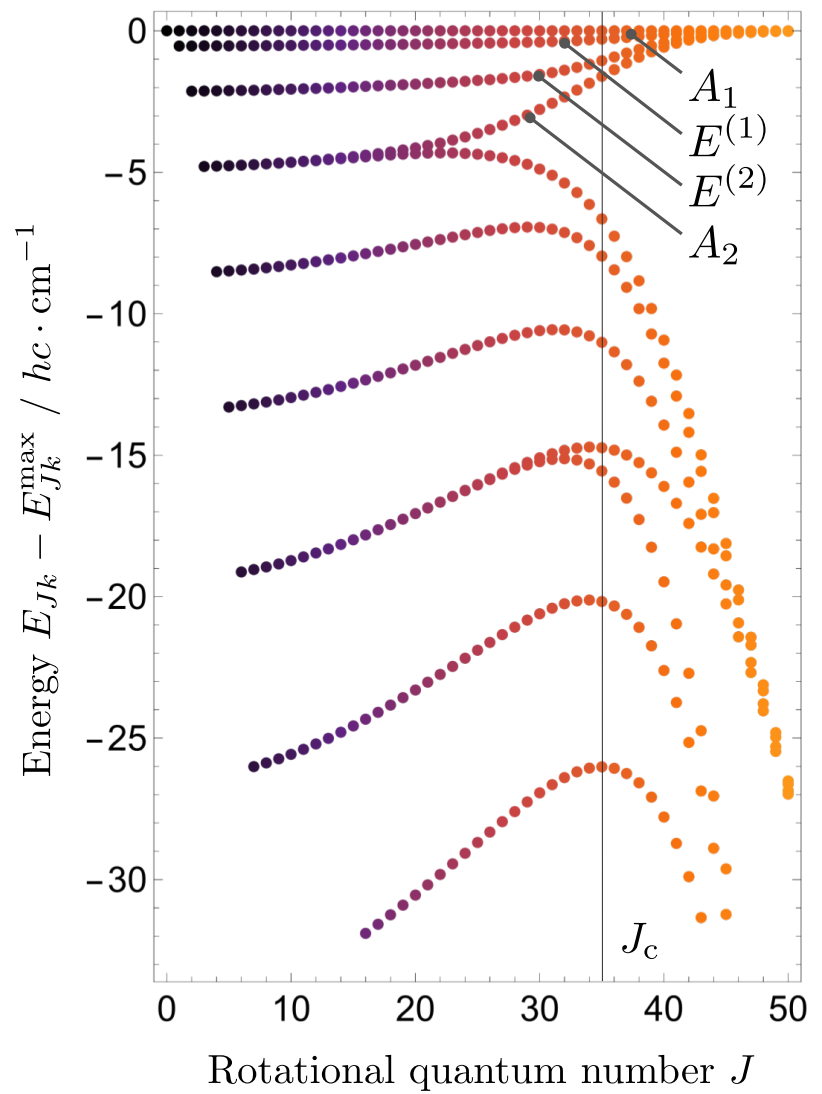

b. Localized chiral cluster states

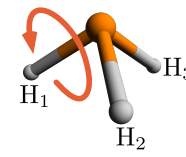

$|1 R\rangle$

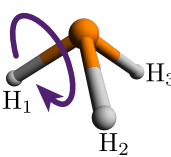

$|1 L\rangle$

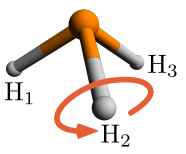

$|2 R\rangle$

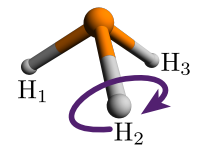

$|2 L\rangle$

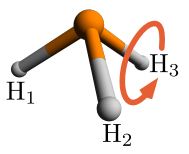

$|3 R\rangle$

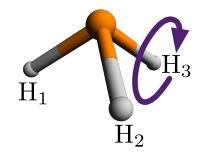

$|3 L\rangle$

\section{c. Delocalized $\mathbf{C}_{3 \mathrm{v}}(\mathrm{M})$ wavefunctions}

$$
\begin{aligned}
& \left|A_{1}\right\rangle=\frac{1}{\sqrt{6}}(|1 R\rangle+|2 R\rangle+|3 R\rangle+|1 L\rangle+|2 L\rangle+|3 L\rangle) \\
& \left|A_{2}\right\rangle=\frac{1}{\sqrt{6}}(|1 R\rangle+|2 R\rangle+|3 R\rangle-|1 L\rangle-|2 L\rangle-|3 L\rangle) \\
& \left|E_{a}^{(1)}\right\rangle=\frac{1}{2 \sqrt{3}}(2|1 R\rangle-|2 R\rangle-|3 R\rangle-|1 L\rangle+2|2 L\rangle-|3 L\rangle) \\
& \left|E_{b}^{(1)}\right\rangle=\frac{1}{2}(|1 L\rangle-|3 L\rangle+|3 R\rangle-|2 R\rangle) \\
& \left|E_{a}^{(2)}\right\rangle=\frac{1}{2 \sqrt{3}}(2|1 L\rangle-|2 L\rangle-|3 L\rangle-|1 R\rangle+2|2 R\rangle-|3 R\rangle) \\
& \left|E_{b}^{(2)}\right\rangle=\frac{1}{2}(|1 R\rangle-|3 R\rangle+|3 L\rangle-|2 L\rangle)
\end{aligned}
$$

FIG. 2. Rotational energy level clustering in the ground vibrational state of $\mathrm{PH}_{3}$, c.f. Fig. 2 of ref. 14. (a) The energy difference $E_{J k}-E_{J k}^{\max }$ was plotted for each rotational energy level $E_{J k}$ relative to the maximum energy $E_{J k}^{\max }$ in its $J$ multiplet. (b) Schematic representation of the localized chiral cluster states, which are separated by high kinetic energy barriers. (c) Wavefunctions of the delocalized rotational cluster states in the representation of the localized chiral cluster states.

of this proposal.

In Fig. 3, the wavepacket population at the end of the centrifuge pulse is plotted along with the rotational probability density function $P(\theta, \chi)=\int \mathrm{d} V \mathrm{~d} \phi \psi(t)^{*} \psi(t) \sin \theta$, which illustrates the orientation of the molecule relative to the possible axes of rotation. Here, $\theta, \chi, \phi$ denote the Euler angles, $\mathrm{d} V$ is the volume element associated with the vibrational coordinates, and $\psi(t)$ is the wavepacket. The dominant contribution of $\sim 25 \%$ to the wavepacket is from the $J=42, m=-42$ energy level of the top cluster state at $7686 \mathrm{~cm}^{-1}$. In $\mathrm{PH}_{3}$, clustering occurs above $J_{\mathrm{c}} \approx 35$ and is related to the six islands that emerge on the rotational density plot seen in Fig. 3 a, which correspond to clockwise $(\theta<\pi / 2)$ and anticlockwise $(\theta>\pi / 2)$ rotation about an axis almost coinciding with one of the $\mathrm{P}-\mathrm{H}$ bonds. Application of a strong dc field in the $-Z$ or $+Z$ direction for the duration of the centrifuge pulse, shown in Fig. $3 \mathrm{~b}$, produces only clockwise $(R)$ or anticlockwise $(L)$ rotating enantiomers, respectively. This is a result of the brute-force orientation of the permanent dipole moment at the time when population is transferred into the cluster states. A detailed analysis of the effect of the dc field shows that, in principle, the field is only necessary during the time when the cluster states are populated, whereas it is a loss channel at the start of the centrifuge, and it does not affect the dynamics once the population is within a well-defined angular momentum enantiomer. In order for the population to be adiabatically transferred from the quasi-free-rotor state to the mixed-parity 'single-enantiomer' cluster state, the dc field splitting must be large in comparison to the traversal time of the $J$ manifold by the centrifuge. Classically speaking, the dc field has to be strong enough to favor one enantiomer over the other when the dipole moment is still nearly perpendicular to the dc field.

This mixed-field enantiomer selectivity allows us to probe the chirality of the $R$ and $L$ enantiomers by measuring, for instance, the free-induction decay [24, 25] by a phase-locked microwave receiver [26], see also further details in the supplemental material [15. As expected, the 


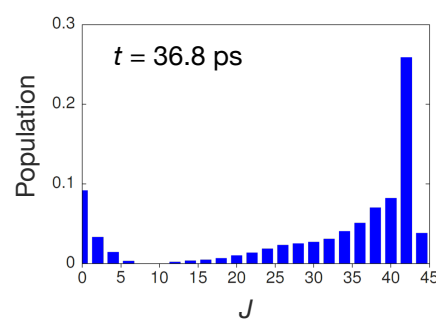

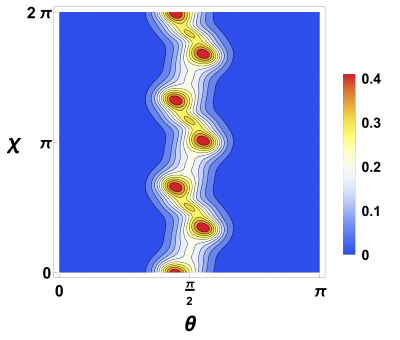

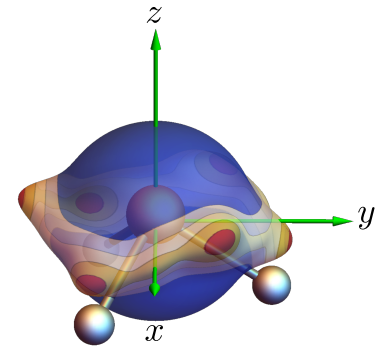

b
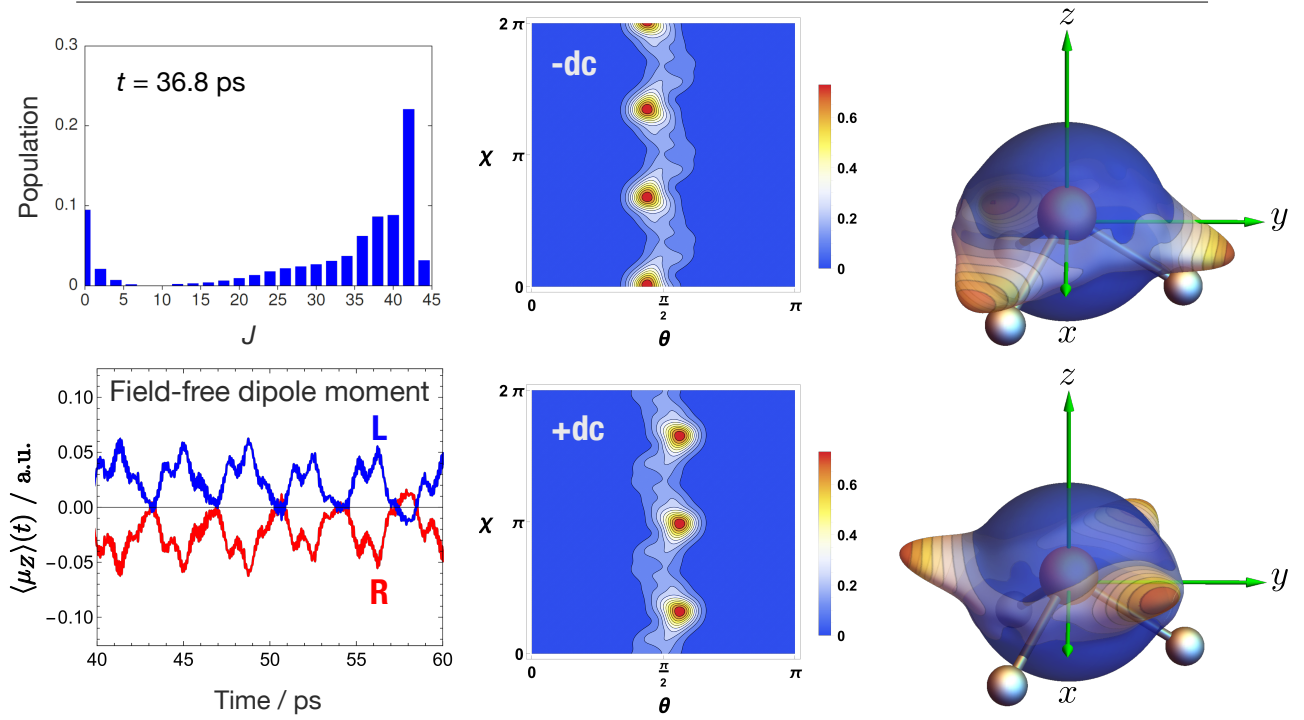

FIG. 3. Wavepacket population by $m=-J$ stationary states and rotational probability density function $P(\theta, \chi)$, in terms of the Euler angles $\theta$ and $\chi$, at the end of the centrifuge pulse $(t=36.8 \mathrm{ps})$, (a) without, and (b) with a dc field of $\pm 1 \mathrm{MV} / \mathrm{cm}$ applied. Wavepacket population of stationary states with odd $J$ quantum numbers or $m \neq-J$ are much smaller and not shown in the plot. The two-dimensional rotational density contour plot, shown in the middle, has been projected onto a sphere, shown on the right hand side, to illustrate the orientation of $\mathrm{PH}_{3}$ and the molecular frame relative to the rotation axes. Each high-density island (red) indicates an axis of rotation and $x, y, z$ refer to the molecule-fixed axis system. In the bottom left hand corner, the time evolution of the expectation value of the laboratory-fixed effective dipole moment $\left\langle\mu_{Z}\right\rangle$ has been calculated for the $R$ and $L$ rotating enantiomers. The exhibited anti-phase behavior between the rotating enantiomers confirms chirality.

time evolution of the permanent electric dipole moment expectation value $\left\langle\mu_{Z}\right\rangle$ in the laboratory-fixed frame shows anti-phase behavior between the rotating enantiomers.

If $\mathrm{PH}_{3}$ is excited above $J=42$, the axis of rotation moves closer to the $\mathrm{P}-\mathrm{H}$ bond 14 . However, our simulations have shown that it is difficult to efficiently transfer population to higher $J$ states, notably across the range $J \approx 40-50$. Instead, wavepacket population gradually spreads across several energy levels and cluster states at each excitation of $J$, resulting in a spectrally broadened rotational wavepacket. For example, if the centrifuge pulse used in this work is left to propagate until $t=48.5 \mathrm{ps}$, only $1 \%$ of population is transferred to the $J=58, m=-58$ energy level of the top cluster state at $14133 \mathrm{~cm}^{-1}$. Clustering represents a significant change in the rotational dynamics and as $\mathrm{PH}_{3}$ rotates faster its structure distorts, leading to the breakdown of $\mathbf{C}_{3 v}(\mathrm{M})$ molecular symmetry. The quantum number $k$ is no longer a good quantum number and the $\Delta k=0$ selection rule that partially governed transitions up to $J_{\mathrm{c}}$ becomes weak. The spreading of wavepacket population can be marginally counteracted with a more tailored centrifuge pulse [27, e.g., non-linear angular acceleration and intensity chirping, however, for the purposes of this study it is enough to show that $\mathrm{PH}_{3}$ is indeed chiral when excited to the $J=42, m=-42$ cluster state.

The concept of true and false chirality was introduced to clarify situations where motion played an essential role 28]. Truly chiral systems possess two distinct enantiomeric states that can be interconverted by space inversion, but not by time reversal combined with any proper spatial rotation. As shown in Fig. 4, if $\mathrm{PH}_{3}$ can be forced to translate along the axis of rotation then the system is truly chiral, i. e., exhibits time-invariant enantiomorphism. If not, the system possesses false chirality, i. e., time-noninvariant enantiomorphism. Distinguishing between true and false chirality has implications for topics such as parity violation [29] and absolute enantioselection [30, and there is still a great deal to learn about this distinction. For example, it was recently reported that 


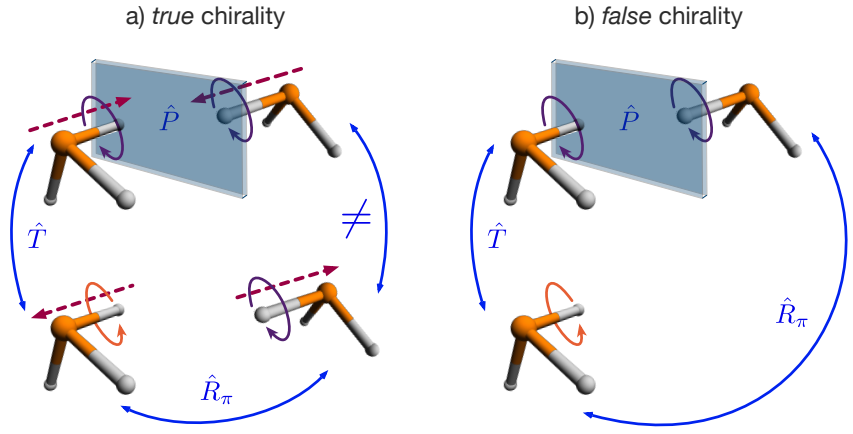

FIG. 4. Definition of true and false chirality. (a) If $\mathrm{PH}_{3}$ is translating along the axis of rotation (dashed arrow), space inversion $(\hat{P})$ is not equivalent to time reversal $(\hat{T})$ combined with any proper spatial rotation $\left(\hat{R}_{\pi}\right)$ and the system is truly chiral. (b) If $\mathrm{PH}_{3}$ is not translating, the system exhibits false chirality as space inversion is equivalent to time reversal combined with spatial rotation.

a falsely chiral influence can generate an enantiomeric excess in a reaction far from equilibrium 31. In $\mathrm{PH}_{3}$ there is an opportunity to further explore the differences between true and false chirality by probing the system with and without translation, or by studying isotopically substituted species such as $\mathrm{PHD}_{2}$, which will possess truly chiral cluster states even when it is not translating along the axis of rotation.

To establish how, if at all, RIC differs from traditional "static" chirality, it will be necessary to study the interaction of the rotating enantiomers with other chiral entities such as light. Achieving a deeper understanding should contribute to the development of chirality-based molecular and material properties, new states of matter, and the potential utilization of RIC in novel metamaterials or optical devices. It is also of interest to image RIC using sensitive techniques such as photoelectron circular dichroism (PECD) 32. It has been shown that changes in the nuclear geometry caused by vibrational motion can reverse the forward-backward asymmetry in the photoelectron angular distribution 33. One can, therefore, expect that PECD measurements of RIC may reveal unexpected behavior. We plan to investigate these topics in future work.

This work has been supported by the Deutsche Forschungsgemeinschaft (DFG) through the excellence cluster "The Hamburg Center for Ultrafast Imaging - Structure, Dynamics and Control of Matter at the Atomic Scale" (CUI, EXC1074) and the priority program 1840 "Quantum Dynamics in Tailored Intense Fields" (QUTIF, KU1527/3), by the European Research Council through the Consolidator Grant COMOTION (ERCKüpper-614507), by the Helmholtz Association "Initiative and Networking Fund", and by the COST action MOLIM (CM1405). A. O. gratefully acknowledges a fellowship from the Alexander von Humboldt Foundation. andrey.yachmenev@cfel.de molecule-imaging.org

[1] P. R. Bunker and P. Jensen, "Chirality in rotational energy level clusters," J. Mol. Spectrosc. 228, 640-644 (2004).

[2] P. Jensen, "Local modes in vibration-rotation spectroscopy," WIREs Comput. Mol. Sci. 2, 494-512 (2012)

[3] T. Kawasaki, Y. Matsumura, T. Tsutsumi, K. Suzuki, M. Ito, and K. Soai, "Asymmetric autocatalysis triggered by carbon isotope $\left({ }^{13} \mathrm{C} /{ }^{12} \mathrm{C}\right)$ chirality," Science 324, 492495 (2009)

[4] A. Matsumoto, H. Ozaki, S. Harada, K. Tada, T. Ayugase, H. Ozawa, T. Kawasaki, and K. Soai, "Asymmetric induction by a nitrogen ${ }^{14} \mathrm{~N} /{ }^{15} \mathrm{~N}$ isotopomer in conjunction with asymmetric autocatalysis," Angew. Chem. Int. Ed. 55, 15246-15249 (2016)

[5] Y. Ohshima and H. Hasegawa, "Coherent rotational excitation by intense nonresonant laser fields," Int. Rev. Phys. Chem. 29, 619-663 (2010)

[6] V. Milner and J. W. Hepburn, "Laser control of ultrafast molecular rotation," Adv. Chem. Phys. 159, 395-412 (2016)

[7] L. Yuan, S. W. Teitelbaum, A. Robinson, and A. S. Mullin, "Dynamics of molecules in extreme rotational states," Proc. Natl. Acad. Sci. U. S. A. 108, 6872-6877 (2011)

[8] A. A. Milner, A. Korobenko, J. W. Hepburn, and V. Milner, "Effects of ultrafast molecular rotation on collisional decoherence," Phys. Rev. Lett. 113, 043005 (2014)

[9] Y. Khodorkovsky, U. Steinitz, J.-M. Hartmann, and I. Sh. Averbukh, "Collisional dynamics in a gas of molecular super-rotors," Nat. Commun. 6, 7791 (2015)

[10] M. J. Murray, H. M. Ogden, C. Toro, Q. Liu, D. A. Burns, M. H. Alexander, and A. S. Mullin, "State-specific collision dynamics of molecular super rotors with oriented angular momentum," J. Phys. Chem. A 119, 12471-12479 (2015)

[11] L. Yuan, C. Toro, M. Bell, and A. S. Mullin, "Spectroscopy of molecules in very high rotational states using an optical centrifuge," Faraday Disc. 150, 101-111 (2011)

[12] A. A. Milner, A. Korobenko, K. Rezaiezadeh, and V. Milner, "From gyroscopic to thermal motion: A crossover in the dynamics of molecular superrotors," Phys. Rev. X 5, 031041 (2015).

[13] J. Karczmarek, J. Wright, P. Corkum, and M. Ivanov, "Optical centrifuge for molecules," Phys. Rev. Lett. 82, 3420-3423 (1999)

[14] S. N. Yurchenko, W. Thiel, S. Patchkovskii, and P. Jensen, "Theoretical evidence for the formation of rotational energy level clusters in the vibrational ground state of $\mathrm{PH}_{3}$," Phys. Chem. Chem. Phys. 7, 573-582 (2005)

[15] See Supplemental Material [insert url], which includes Refs. $34 \mid 40$

[16] S. N. Yurchenko, W. Thiel, and P. Jensen, "Theoretical ROVibrational energies (TROVE): A robust numerical approach to the calculation of rovibrational energies for polyatomic molecules," J. Mol. Spectrosc. 245, 126-140 (2007).

[17] A. Yachmenev and S. N. Yurchenko, "Automatic differentiation method for numerical construction of the rotationalvibrational Hamiltonian as a power series in the curvilinear internal coordinates using the Eckart frame," J. Chem. 
Phys. 143, 014105 (2015)

[18] S. N. Yurchenko, A. Yachmenev, and R. I. Ovsyannikov, "Symmetry adapted ro-vibrational basis functions for variational nuclear motion calculations: TROVE approach," J. Chem. Theory Comput. 13, 4368 (2017).

[19] C. Sousa-Silva, A. F. Al-Refaie, J. Tennyson, and S. N. Yurchenko, "ExoMol line lists - VII. The rotationvibration spectrum of phosphine up to $1500 \mathrm{~K}$," Mon. Not. R. Astron. Soc. 446, 2337-2347 (2015).

[20] A. Owens and A. Yachmenev, "RichMol: A general variational approach for rovibrational molecular dynamics in external electric fields," J. Chem. Phys. 148, 124102 (2018).

[21] Y.-P. Chang, D. A. Horke, S. Trippel, and J. Küpper, "Spatially-controlled complex molecules and their applications," Int. Rev. Phys. Chem. 34, 557-590 (2015) arXiv:1505.05632 [physics]

[22] D. A. Horke, Y.-P. Chang, K. Długołęcki, and J. Küpper, "Separating para and ortho water," Angew. Chem. Int. Ed. 53, 11965-11968 (2014), arXiv:1407.2056 [physics]

[23] Y. Ishida and S. Hiyama, "Production of high Lo Surdo fields," Nature 124, 129-129 (1929)

[24] D. Patterson, M. Schnell, and J. M. Doyle, "Enantiomerspecific detection of chiral molecules via microwave spectroscopy," Nature 497, 475-477 (2013).

[25] A. Yachmenev and S. N. Yurchenko, "Detecting chirality in molecules by linearly polarized laser fields," Phys. Rev. Lett. 117, 033001 (2016)

[26] J. Kim, J. A. Cox, J. Chen, and F. X. Kärtner, "Drift-free femtosecond timing synchronization of remote optical and microwave sources," Nat. Photon. 2, 733-736 (2008)

[27] A. Owens, A. Yachmenev, and J. Küpper, "Coherent control of the rotation axis of molecular superrotors," J. Phys. Chem. Lett. 9, 4206-4209 (2018), arXiv:1807.04016 [physics]

[28] L. D. Barron, Molecular Light Scattering and Optical Activity, 2nd ed. (Cambridge University Press, Cambridge, 2004).

[29] L. D. Barron, "True and false chirality and parity violation," Chem. Phys. Lett. 123, 423-427 (1986)

[30] L. D. Barron, "True and false chirality and absolute asymmetric synthesis," J. Am. Chem. Soc. 108, 5539-5542 (1986)

[31] N. Micali, H. Engelkamp, P. G. van Rhee, P. C. M. Christianen, L. M. Scolaro, and J. C. Maan, "Selection of supramolecular chirality by application of rotational and magnetic forces," Nat. Chem. 4, 201-207 (2012).

[32] M. H. M. Janssen and I. Powis, "Detecting chirality in molecules by imaging photoelectron circular dichroism," Phys. Chem. Chem. Phys. 16, 856-871 (2014)
[33] G. A. Garcia, L. Nahon, S. Daly, and I. Powis, "Vibrationally induced inversion of photoelectron forwardbackward asymmetry in chiral molecule photoionization by circularly polarized light," Nat. Commun. 4, 2132 (2013).

[34] K. Aidas, C. Angeli, K. L. Bak, V. Bakken, R. Bast, L. Boman, O. Christiansen, R. Cimiraglia, S. Coriani, P. Dahle, E. K. Dalskov, U. Ekström, T. Enevoldsen, J. J. Eriksen, P. Ettenhuber, B. Fernández, L. Ferrighi, H. Fliegl, L. Frediani, K. Hald, A. Halkier, C. Hättig, H. Heiberg, T. Helgaker, A. C. Hennum, H. Hettema, E. Hjertenæs, S. Høst, I.-M. Høyvik, M. F. Iozzi, B. Jansík, H. J. Aa. Jensen, D. Jonsson, P. Jørgensen, J. Kauczor, S. Kirpekar, T. Kjærgaard, W. Klopper, S. Knecht, R. Kobayashi, H. Koch, J. Kongsted, A. Krapp, K. Kristensen, A. Ligabue, O. B. Lutnæs, J. I. Melo, K. V. Mikkelsen, R. H. Myhre, C. Neiss, C. B. Nielsen, P. Norman, J. Olsen, J. M. H. Olsen, A. Osted, M. J. Packer, F. Pawlowski, T. B. Pedersen, P. F. Provasi, S. Reine, Z. Rinkevicius, T. A. Ruden, K. Ruud, V. V. Rybkin, P. Sałek, C. C. M. Samson, A. S. de Merás, T. Saue, S. P. A. Sauer, B. Schimmelpfennig, K. Sneskov, A. H. Steindal, K. O. Sylvester-Hvid, P. R. Taylor, A. M. Teale, E. I. Tellgren, D. P. Tew, A. J. Thorvaldsen, L. Thøgersen, O. Vahtras, M. A. Watson, D. J. D. Wilson, M. Ziolkowski, and H. Ågren, "The Dalton quantum chemistry program system," WIREs Comput. Mol. Sci. 4, 269-284 (2014).

[35] S. N. Yurchenko, R. J. Barber, A. Yachmenev, W. Thiel, P. Jensen, and J. Tennyson, "A variationally computed $T=300 \mathrm{~K}$ line list for $\mathrm{NH}_{3}$," J. Phys. Chem. A 113, 11845-11855 (2009)

[36] R. B. Sidje, "Expokit: a software package for computing matrix exponentials," ACM Trans. Math. Soft. 24, 130156 (1998)

[37] W. G. Harter and C. W. Patterson, "Rotational energy surfaces and high- $J$ eigenvalue structure of polyatomic molecules," J. Chem. Phys. 80, 4241-4261 (1984)

[38] J. S. Kienitz, K. Długołęcki, S. Trippel, and J. Küpper, "Improved spatial separation of neutral molecules," J. Chem. Phys. 147, 024304 (2017), arXiv:1704.08912 |physics|

[39] J. S. Kienitz, Orientation of state selected OCS molecules in mixed strong dc and laser fields, Dissertation, Universität Hamburg, Hamburg, Germany (2016).

[40] I. Tutunnikov, E. Gershnabel, S. Gold, and I. Sh. Averbukh, "Selective orientation of chiral molecules by laser fields with twisted polarization," J. Phys. Chem. Lett. 9, 1105-1111 (2018) 